\title{
Reactivity in interstellar ice analogs: role of the structural evolution
}

\author{
P. Ghesquière ${ }^{1, \star}$, A. Ivlev ${ }^{2}$, J. A. Noble ${ }^{3, \star \star}$, and P. Theulé ${ }^{3}$ \\ ${ }^{1}$ Laboratoire Univers et Particules de Montpellier, UMR 5299, CNRS et Université de Montpellier, Place Eugène Bataillon, \\ 34095 Montpellier Cedex 05, France \\ 2 Max-Planck-Institut für Extraterrestrische Physik, Giessenbachstr. 1, 85748 Garching, Germany \\ 3 Aix-Marseille Université, Physique des Interactions Ioniques et Moléculaires, PIIM UMR CNRS 7345, Avenue Escadrille \\ Normandie-Niémen, 13397 Marseille Cedex 20, France, \\ e-mail: patrice.theule@univ-amu.fr
}

Received 13 November 2017 / Accepted 5 February 2018

\begin{abstract}
Context. The synthesis of interstellar complex organic molecules in ice involves several types of reactions between molecules and/or radicals that are usually considered to be diffusion controlled.

Aims. We aim to understand the coupling between diffusion and reactivity in the interstellar ice mantle using a model binary reaction in the diffusion-limited regime.

Methods. We performed isothermal kinetic laboratory experiments on interstellar ice analogs at low temperatures, using the $\mathrm{NH}_{3}: \mathrm{CO}_{2}: \mathrm{H}_{2} \mathrm{O}$ model system where reactants $\mathrm{NH}_{3}$ and $\mathrm{CO}_{2}$ have a low reaction barrier and are diluted in a water-dominated ice.

Results. We found that in the diffusion-limited regime, the reaction kinetics is not determined by the intrinsic bulk diffusivity of reactants. Instead, reactions are driven by structural changes evolving in amorphous water ice, such as pore collapse and crystallization. Diffusion of reactants in this case likely occurs along the surface of (tiny) cracks generated by the structural changes.

Conclusions. The reactivity driven by the structural changes breaks the conventional picture of reactant molecules/radicals diffusing in a bulk water ice. This phenomenon is expected to lead to a dramatic increase in production rates of interstellar complex organic molecules in star-forming regions.
\end{abstract}

Key words. astrochemistry - molecular processes - ISM: molecules

\section{Introduction}

Many interstellar complex organic molecules (iCOMs) are thought to form in the interstellar ice mantles (Aikawa et al. 2003; Caselli \& Ceccarelli 2012). The detection of iCOMs in the interstellar medium (ISM) has been accelerated the past decades thanks to the recent availability of powerful instruments such as Herschel or ALMA (Belloche et al. 2016). The detection of iCOMs in our solar system by recent missions such as Rosetta (Quirico et al. 2016) or New Horizon (Gladstone et al. 2016), as well as the discovery of exoplanet atmospheres (Swain et al. 2009), poses a very important question about the level of molecular complexity that can be reached in space.

The low-temperature solid-state chemistry that leads to the formation of iCOMs in ice is poorly known Bacmann et al. 2012. It occurs in amorphous ice, also called amorphous solid water (ASW) ice, which is observed both in dense molecular clouds as a frost on interstellar dust (Gibb et al. 2004) and as a cometary ice (Davies et al. 1997). This chemistry is out-ofequilibrium and therefore kinetically controlled. A set of radicalradical (Zhitnikov \& Dmitriev 2002), radical-molecule (Oba et al.

^ Present address: Institut de Chimie Moléculaire de l'Université de Bourgogne, UMR 6302 CNRS, Université de Bourgogne FrancheComté, 9 Avenue A. Savary, BP 47 870, 21078 Dijon Cedex, France.

$\star \star$ Present address: Laboratoire de Physique des Lasers, Atomes et Molécules, UMR CNRS 8523, Université Lille 1 Sciences Technologies, Villeneuve d'Ascq Cedex, France.
2012; Borget et al. 2017), and molecule-molecule (Theulé et al. 2013) reactions takes place in the ice, with characteristic reaction barriers on the order of 0,1 , and $10 \mathrm{~kJ} \mathrm{~mol}^{-1}$ respectively, which are typically lower than the diffusion barriers of the reactants (Livingston et al. 2002). Ice chemistry is therefore largely diffusion limited. The surface diffusion is known to be much faster than the bulk diffusion (see., e.g., Livingston et al. 2002). On the other hand, the surface chemistry only affects the topmost monolayer (ML), that is, 2-3\% of a typical 100 ML thick ice, and thus 97$98 \%$ of the reactants remain in the bulk once the mantle is built up. In the conventional picture of reactants diffusing in the ice mantle and meeting each other to form iCOMs, the bulk chemistry at low or mild (before the ice desorption) temperatures should be inefficient because of the scarcity of the reactants and the slowness of their bulk diffusion. However, our previous works on diffusion of solute molecules in bulk ice show a water solvent-driven diffusion (Mispelaer et al. 2013; Ghesquière et al. 2015), which questions this conventional picture.

In this work we experimentally address the kinetics of binary reactions occurring in the bulk ice in the diffusion-limited regime. The characteristic diffusion time that is longer than the reaction time is ensured by a low reaction barrier and by a high dilution of solute reactants in water ice (the latter reduces the probability that reactions occur due to accidental closeness of the reactants). Experimentally, it is obviously easier to handle closed-shell molecules than radicals, and therefore we chose the model reaction $\mathrm{CO}_{2}+2 \mathrm{NH}_{3} \rightarrow \mathrm{NH}_{4}^{+} \mathrm{NH}_{2} \mathrm{COO}^{-}$, where one molecule of $\mathrm{CO}_{2}$ reacts with two molecules 
of $\mathrm{NH}_{3}$ (ternary reaction) to form $\mathrm{NH}_{4}^{+} \mathrm{NH}_{2} \mathrm{COO}^{-}$ammonium carbamate (AC). The measure barrier for this reaction, $\approx 5 \mathrm{~kJ} \mathrm{~mol}^{-1}$ (Noble et al. 2014), is lower than the bulk diffusion barriers, which is expected to be about a few tens of $\mathrm{kJ} \mathrm{mol}^{-1}$ (Livingston et al. 2002; Smith et al. 1997a). The dilution of both $\mathrm{CO}_{2}$ and $\mathrm{NH}_{3}$ reactants was chosen to exceed 1:4 (with respect to water), since neutron beam scattering experiments show that one molecule on average has 4.2 nearest neighbors in ASW (Bowron et al. 2006; Finney 2002).

Our experimental kinetic studies on this model reaction show that the consumption of the reactants and apparition of products is intimately correlated with the structural changes evolving in ASW ices. This breaches the classical picture of reactants undergoing a bulk diffusion in the ice mantles. The reactivity mechanism driven by structural changes in the bulk ice has strong astrophysical implications as it allows radical-radical, radical-molecule, and molecule-molecule reactions to occur at a rate much higher than the previously expected bulk diffusion rate. This phenomenon should stimulate production of iCOMs in star-forming regions, where the ice is warmed up, and therefore it has to be taken into account when modeling iCOM observations.

\section{Experimental}

Our experiments were carried out on the reactivity on interstellar grains (RING) experimental setup, which has been especially designed to study chemistry in interstellar ice analogs. A goldplated copper surface was maintained at a low temperature using a closed-cycle helium cryostat (ARS Cryo, model DE-204 $\mathrm{SB}, 4 \mathrm{~K}$ cryogenerator) within a high-vacuum chamber a at a few $10^{-9} \mathrm{hPa}$ base pressure (at room temperature). The surface temperature was controlled using a Lakeshore Model 336 temperature controller and a cylindrical heating resistance mounted inside the copper sample holder. The temperature was measured with a DTGS 670 silicon diode with a $0.1 \mathrm{~K}$ uncertainty. By applying Fourier-transform infrared (FTIR) spectroscopy, absorption spectra were obtained in the reflection mode with a Vertex 70 spectrometer, using either a DTGS detector or a liquid $\mathrm{N}_{2}$ cooled MCT detector (Mispelaer et al. 2013). A typical spectrum has a $1 \mathrm{~cm}^{-1}$ resolution and is averaged over 100 interferograms. Gas-phase $\mathrm{CO}_{2}$ and $\mathrm{NH}_{3}$ are commercially available in the form of $99.9995 \%$ pure gas from Air Liquide. The $\mathrm{H}_{2} \mathrm{O}$ vapor was obtained from deionized water that was purified by several freeze-pump-thaw cycles, carried out under primary vacuum. A gas mixture of $\mathrm{NH}_{3}, \mathrm{CO}_{2}$, and $\mathrm{H}_{2} \mathrm{O}$ was prepared in a $1 \mathrm{~L}$ glass vacuum line, where it was kept for one hour to ensure a homogeneous gas mixing.

A compact $\mathrm{NH}_{3}: \mathrm{CO}_{2}: \mathrm{H}_{2} \mathrm{O}$ cryogenic molecular solid was formed by low-pressure vapor deposition of the gas mixture at normal incidence on the surface at $80 \mathrm{~K}$. This temperature was chosen to have a (partially) compact ASW ice while freezing $\mathrm{CO}_{2}$, which is known to desorb at $95 \mathrm{~K}$ (Noble et al. 2012). The initial ice thickness was estimated using the IR absorption band of the water $\mathrm{OH}$ stretching (band strength $2.1 \times 10^{-16} \mathrm{~cm} \mathrm{~mol}^{-1}$ Hagen et al. 1983) at $3280 \mathrm{~cm}^{-1}$ and adopting the ASW ice density of $0.94 \mathrm{~g} \mathrm{~cm}^{-3}$ (Mayer \& Pletzer 1986). The initial $\mathrm{NH}_{3}: \mathrm{CO}_{2}: \mathrm{H}_{2} \mathrm{O}$ concentration ratio in ice was deduced from the IR spectrum as illustrated in Fig. 1. The uncertainty for the concentrations and the thicknesses is estimated to be about $20 \%$, which is primarily determined by uncertainties for the band strengths and the ice density. Then, to prevent $\mathrm{NH}_{3}$ and $\mathrm{CO}_{2}$ desorption, a "cork" of pure water ice (of a few tens of layers) was deposited on top of the initial ice mixture. According to Fraser et al. (2001), it takes about three days to desorb such a

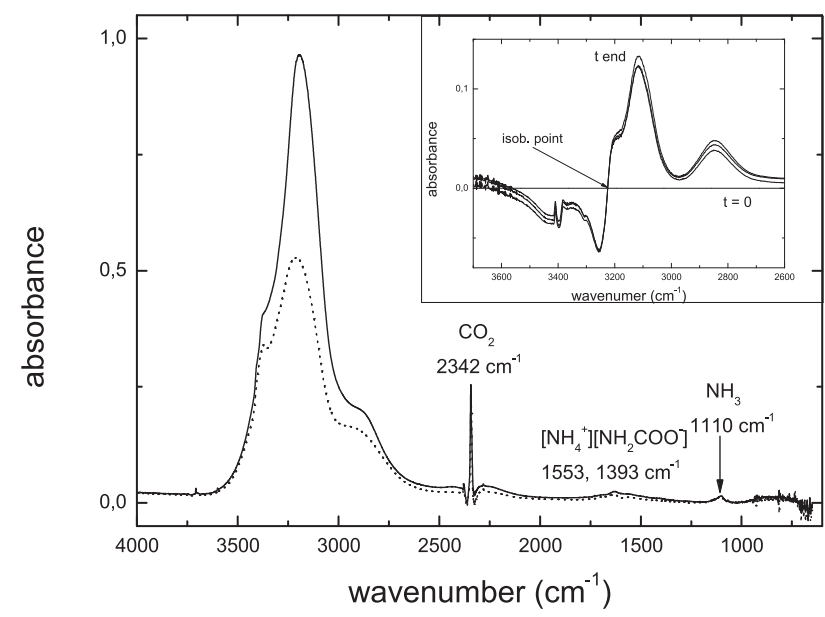

Fig. 1. FTIR spectrum of an $\mathrm{NH}_{3}: \mathrm{CO}_{2}: \mathrm{H}_{2} \mathrm{O}$ ice mixture at $80 \mathrm{~K}$ (expt 11) after the deposition (dotted lines) and after $\mathrm{a}_{2} \mathrm{O}$ "cork" is placed on top of it (solid lines). The inset illustrates the structural evolution of ASW ice, characterized by the difference spectrum of the water ice stretching mode at constant temperature. The isobestic point at $3225 \mathrm{~cm}^{-1}$ is not affected by the evolution.

cork at $130 \mathrm{~K}$. Hence, the cork ensured that the observed disappearance of the reactants at all temperatures was solely due to their reactivity.

The initial ASW ice (with a cork on it) was left to relax at $100 \mathrm{~K}$ for one hour to stabilize the pore network (Wu et al. 2011; Mispelaer et al. 2013; Mitchell et al. 2016) and thus to decrease the surface-to-volume ratio (Bossa et al. 2014). Working with a relaxed ice stabilizes the pore clustering and thus should improve the reproducibility of our experiments. However, Isokoski et al. (2014) showed that even after annealing at $120 \mathrm{~K}$, the residual porosity remains at $17 \pm 3 \%$, which has been corroborated by Mitchell et al. (2016) and Hill et al. (2016). This means that even after one hour of relaxing at $100 \mathrm{~K}$, our ice mixture may not be fully compact, and porosity is expected to affect the crystallization process (Mitchell et al. 2016). The ice was then heated to the desired temperature and kept at this level for the duration of the experiment. Table 1 summarizes the experiments performed with different $\mathrm{NH}_{3}: \mathrm{CO}_{2}: \mathrm{H}_{2} \mathrm{O}$ initial ratios, thicknesses, and temperatures. Experiments at $120 \mathrm{~K}$ are limited by the maximum time of stability of our experimental setup, while at $140 \mathrm{~K}$, they are limited by the desorption time.

The diffusion-reaction isothermal kinetics is measured by monitoring the evolution of the reactants and products abundances, deduced from their characteristic IR absorption bands: the asymmetric stretching band of $\mathrm{CO}_{2}$ at $2342 \mathrm{~cm}^{-1}$ (band strength $1.4 \times 10^{-17} \mathrm{~cm} \mathrm{~mol}^{-1}$ in water ice Sandford \& Allamandola 1990), the $\mathrm{NH}_{3}$ umbrella mode at $1110 \mathrm{~cm}^{-1}$ (band strength $1.3 \times 10^{-17} \mathrm{~cm} \mathrm{~mol}^{-1}$ Kerkhof et al. 1999), as well as the $v_{a s}\left(\mathrm{COO}^{-}\right)\left[\mathrm{NH}_{4}^{+}\right]\left[\mathrm{NH}_{2} \mathrm{COO}^{-}\right]$band at $1553 \mathrm{~cm}^{-1}$ and the $v(\mathrm{CN})$ $\left[\mathrm{NH}_{4}^{+}\right]\left[\mathrm{NH}_{2} \mathrm{COO}^{-}\right]$band at $1393 \mathrm{~cm}^{-1}$ for ammonium carbamate (AC) (Bossa et al. 2008). While for different isothermal experiments between $120 \mathrm{~K}$ and $140 \mathrm{~K}$ the disappearance of the $\mathrm{CO}_{2}$ reactant and the appearance of the $\mathrm{AC}$ product yield clear curves, the time evolution of $\mathrm{NH}_{3}$ is hard to record because of the broadness of the $1110 \mathrm{~cm}^{-1}$ band and the noise from our detector in this region.

The structural changes evolving in the ice are quantified with the difference spectrum in the $\mathrm{OH}$ stretching mode region: we monitored the maximum at $2900-3200 \mathrm{~cm}^{-1}$ and the minimum at $3250-3400 \mathrm{~cm}^{-1}$. The former spectral region is affected by 
Table 1. List of experiments with the corresponding temperature, initial concentration ratio, and thickness.

\begin{tabular}{ccccccc}
\hline \hline Experiment & $T(\mathrm{~K})$ & $\mathrm{NH}_{3}: \mathrm{CO}_{2}: \mathrm{H}_{2} \mathrm{O}$ & Thickness $(\mathrm{nm})$ & $\tau_{\mathrm{SE}}(\mathrm{s})$ & $\tau_{\mathrm{CO}_{2}}(\mathrm{~s})$ & $\tau_{\mathrm{AC}}(\mathrm{s})$ \\
\hline 1 & 120 & $15: 8: 100$ & 348 & $5.7 \times 10^{4}$ & $1.1 \times 10^{5}$ & $7.0 \times 10^{4}$ \\
2 & 120 & $16: 8: 100$ & 160 & $2.2 \times 10^{4}$ & $1.9 \times 10^{4}$ & $1.9 \times 10^{4}$ \\
3 & 125 & $8: 5: 100$ & 258 & $1.7 \times 10^{4}$ & $1.7 \times 10^{4}$ & $3.8 \times 10^{4}$ \\
4 & 130 & $31: 4: 100$ & 338 & $1.9 \times 10^{4}$ & $2.3 \times 10^{4}$ & - \\
5 & 130 & $21: 8: 100$ & 296 & $2.1 \times 10^{4}$ & $1.9 \times 10^{4}$ & $3.4 \times 10^{4}$ \\
6 & 130 & $16: 9: 100$ & 218 & $1.9 \times 10^{4}$ & $2.1 \times 10^{4}$ & $7.4 \times 10^{4}$ \\
7 & 130 & $5: 2: 100$ & 228 & $3.2 \times 10^{4}$ & $1.5 \times 10^{4}$ & $4.8 \times 10^{4}$ \\
8 & 135 & $15: 8: 100$ & 269 & $1.3 \times 10^{4}$ & $1.5 \times 10^{4}$ & $1.1 \times 10^{4}$ \\
9 & 135 & $7: 6: 100$ & 217 & $2.2 \times 10^{3}$ & $3.0 \times 10^{3}$ & $2.5 \times 10^{4}$ \\
10 & 140 & $21: 8: 100$ & 332 & $1.4 \times 10^{4}$ & $5.7 \times 10^{3}$ & $1.2 \times 10^{4}$ \\
11 & 140 & $17: 6: 100$ & 307 & $2.0 \times 10^{4}$ & $4.5 \times 10^{3}$ & $1.6 \times 10^{4}$ \\
12 & 140 & $15: 5: 100$ & 191 & $5.2 \times 10^{3}$ & $2.9 \times 10^{3}$ & $2.9 \times 10^{4}$ \\
13 & 140 & $6: 9: 100$ & 181 & $3.4 \times 10^{3}$ & $1.7 \times 10^{3}$ & $1.1 \times 10^{3}$ \\
\hline
\end{tabular}

Notes. The structural evolution (SE) timescale as well as the reaction timescales for the $\mathrm{CO}_{2}$ reactant and ammonium carbamate (AC) product are also given. We estimate a $20 \%$ uncertainty on the concentration ratio and thicknesses, a $30 \%$ uncertainty on the $\mathrm{SE}$ and $\mathrm{CO}_{2}$ timescales, and up to $100 \%$ uncertainty on the AC timescale.

the profile modification due to $\mathrm{HOH} . . . \mathrm{NH}_{3}$ complexing, while the latter region is affected by the ammonia $\mathrm{NH}$ stretch (Hagen et al. 1983) and possible signal saturation. For these reasons, we followed fixed frequency points instead of integrating bands in the regions, similar to Smith et al. (2011). The isobestic point of each curve, at $3225 \mathrm{~cm}^{-1}$ in the inset in Fig. 1, was not affected by the structural changes. Each spectrum was scaled to the initial spectrum (at $t=0$ ) at the isobestic point, to correct for small but noticeable water desorption from the cork. The deduced structural evolution encompasses different phenomena with different timescales and temperature ranges.

\section{Results}

We performed kinetic experiments for which the disappearance of the $\mathrm{CO}_{2}$ reactant, the appearance of the $\mathrm{AC}$ product, and the structural changes in the ASW sample were recorded as functions of time at a fixed temperature. The results are illustrated in Fig. 2, where all curves are normalized to unity at the maximum; the decreasing $\mathrm{CO}_{2}$ curve is flipped with respect to unity to facilitate the comparison. The results for $120 \mathrm{~K}$ indicate that the reactivity evolves much faster than the expected crystallization because the crystallization induction time in this case $\left(\sim 10^{5} \mathrm{~s}\right.$ according to Faure et al. 2015) exceeds the time of the experiment. This trend is observed in both experiments 1 and 2 . At the same time, the reactivity curve follows the curve of the structural evolution. Given the $17 \pm 3 \%$ residual porosity estimated by Isokoski et al. (2014) after an annealing at $120 \mathrm{~K}$, the structural changes in our case can be entirely attributed to ongoing pore collapse. At $130 \mathrm{~K}$, the induction is likely manifested by a kink seen in the structural evolution curve at $\sim 10^{4} \mathrm{~s}$, which is consistent with the induction time measured for this temperature by Faure et al. (2015) (it is generally expected that the induction time depends on the host species presented in the ice). Again, we see correlated changes occurring in the reactivity and structure of the ice. At $135 \mathrm{~K}$ the predicted induction time becomes quite short, $\sim 3 \times 10^{3} \mathrm{~s}$ (Faure et al. 2015), while at $140 \mathrm{~K}$ it is negligibly short. Hence, unlike the case of $120 \mathrm{~K}$, for experiments at 130,135 , and $140 \mathrm{~K}$, crystallization is expected to be the leading mechanism of the structural evolution. Still, at all
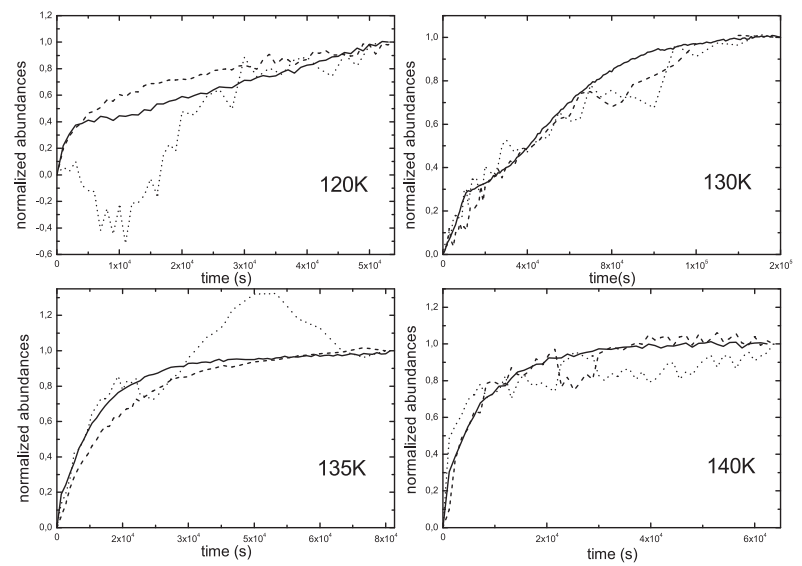

Fig. 2. Kinetics of the decay of the $\mathrm{CO}_{2}$ reactant (band at $2342 \mathrm{~cm}^{-1}$, flipped for a better comparison, dashed line), formation of the AC product (band at $1553 \mathrm{~cm}^{-1}$, dotted line), and structural evolution of ASW ice (point at $3307 \mathrm{~cm}^{-2}$, solid line) at $120 \mathrm{~K}$ (expt 2), $130 \mathrm{~K}$ (expt 7), $135 \mathrm{~K}$ (expt 8), and $140 \mathrm{~K}$ (expt 10).

temperatures we clearly observe a strong correlation between the reaction kinetics and the structural changes, regardless of the leading mechanism behind the latter process.

To quantify this correlation better, we fit the reaction and structural curves against an empirical $1-\exp \left(-t / \tau_{k}\right)$ function, and evaluated the resulting characteristic time $\tau_{k}$ for the whole set of experiments summarized in Table 1 (where $k$ stands for SE, $\mathrm{CO}_{2}$, or $\mathrm{AC}$ ). The uncertainty on the structural evolution (SE) and $\mathrm{CO}_{2}$ timescales is estimated at $30 \%$ and at up to $100 \%$ on the AC timescale. The uncertainties mainly come from the choice of the boundary conditions, from the desorption correction, and from the smallness of band integral variations compared to the baseline changes. Since the $\mathrm{CO}_{2}$ is consumed at the same rate as the carbamate is produced, the apparent difference between their kinetics clearly reflects difficulties of monitoring the evolution of different bands.

The characteristic times for the whole set of experiments are plotted in Fig. 3 versus the temperature. The temperature dependencies for the $\mathrm{CO}_{2}$ consumption and $\mathrm{AC}$ production follow, 


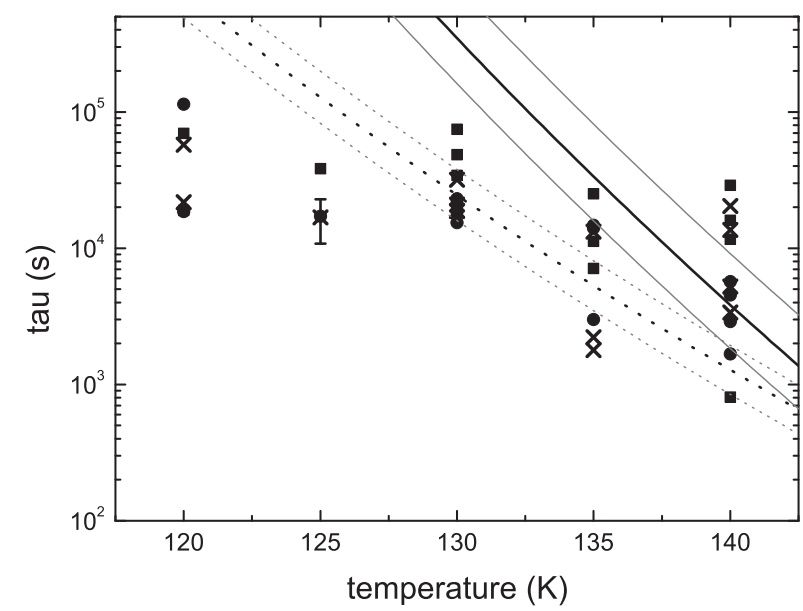

Fig. 3. Comparison of different characteristic timescales, plotted vs. temperature for the experiments listed in Table 1: $\mathrm{CO}_{2}$ reactant decay (circle), AC production (square), and ASW structural evolution (cross). The typical uncertainty on each timescale is illustrated for $T=125 \mathrm{~K}$ (the uncertainty is similar for other temperatures). The crystallization timescales derived from measurements by Faure et al. (2015; dashed line) and Smith et al. (2011; solid line) are also depicted, along with their respective uncertainties.

within the experimental dispersion, the dependence for the ASW structural evolution. The structural evolution times for $130 \mathrm{~K}$, $135 \mathrm{~K}$, and $140 \mathrm{~K}$ are comparable to the crystallization times derived by Smith et al. (2011) and Faure et al. (2015); note that these authors fit their kinetics data with an Avrami law (while we used an exponential law). On the other hand, the characteristic times for $120 \mathrm{~K}$ and $125 \mathrm{~K}$ are substantially shorter than the crystallization times, presumably because the pore collapse becomes the dominant process governing the structural evolution at lower temperatures. Figure 3 also illustrates the spread of timescales between the experiments at a given temperature. Such a large dispersion is typical for amorphous solids and for ASW crystallization in the presence of impurities (Johari 1991; Faure et al. 2015).

\section{Discussion}

The experiments presented above demonstrate a strong correlation between the reaction kinetics in bulk ices and their structural evolution. The observed behavior is very different from a classical picture of reactants diffusing through a bulk network of fixed hydrogen-bonded water molecules, until they occasionally meet to form the reaction products. The results would rather be interpreted as an effect of emerging structural features (such as crack opening) induced in the ASW mantle by crystallization or pore collapse. We point out that structural features are generally known to critically affect transport properties of solids: for instance, diffusion occurring in alloys along microstructural defects, such as grain boundaries or dislocation pipes, can be dramatically faster than bulk diffusion through a monocrystal (Stechauner \& Kozeschnik 2014).

ASW ice is a kinetically metastable solid phase, slowly $(\simeq 10$ million years at $80 \mathrm{~K}$; Baragiola 2003) transforming into thermodynamically stable crystalline ice. During the crystallization process, cracks are formed throughout the ice that suddenly increase the inner surface-to-volume ratio (May et al. 2012). The cracks may span the entire volume of the ice, to form percolating pathways. A part of these cracks open to an outer surface, leading to an episodic release of trapped gases (Smith et al. 1997b; May et al. 2013). The pathways of inner cracks give rise to a long-range diffusion throughout their surface. This suggests that ASW exhibits liquid-like translational diffusion, $\sim 10^{6 \pm 1}$ greater than the bulk diffusion in crystalline ice (Smith et al. 1997a). The hypothesis that the enhanced reactivity is due to surface diffusion along the inner crack walls is further supported by the agreement between the values of $\mathrm{CO}$ diffusion coefficient measured at temperatures where ASW ice is still porous (Mispelaer et al. 2013; Karssemeijer et al. 2014; Lauck et al. 2015) and the surface diffusion calculations (Karssemeijer et al. 2014).

Structural evolution of ASW ice is a complex process. It has been shown that the crystallization has two distinct stages, induction and propagation (May et al. 2013; Faure et al. 2015; Mitchell et al. 2016), and that the initial ice porosity (external and internal) has a major influence on the crystallization kinetics (Mitchell et al. 2016), which, in turn, is affected by the presence of impurities (volatiles) in a water-dominated ice (Johari 1991; Faure et al. 2015). Moreover, although microporosity disappears during the pore collapse and the resulting ice compaction (Isokoski et al. 2014), mesoporosity (2-50 nm) may still be present at $140 \mathrm{~K}$ (Raut et al. 2007).

The conclusion that molecular transport in ASW ice can be driven by ongoing structural changes has several implications of general importance:

1. The reactivity of diluted molecules/radicals in water ice can be dramatically enhanced compared to the case when their transport is governed by a regular bulk diffusion.

2. The diffusion throughout the surface of cracks created in ASW ice is expected to be much less dependent on a particular sort of (heavy) molecules/radicals (Mispelaer et al. 2013; Ghesquière et al. 2015).

3. Volatile molecules/radicals that do not occur at the surface of the cracks remain trapped in the ice (Collings et al. 2004; Viti et al. 2004; May et al. 2013). This affects the overall reaction yield as well as the release of volatiles from the ice (May et al. 2013). The yield is expected to depend on a number of parameters, such as the ice thickness, temperature increase rate, initial porosity, and thermal history.

\section{Astrophysical relevance and conclusions}

The presented results may have profound astrochemical consequences, changing traditional theoretical description of reactiondiffusion in gas-grain chemistry models (see., e.g., Tielens \& Hagen 1982; Hasegawa et al. 1992). The results are particularly relevant to star-forming regions (Caselli \& Ceccarelli 2012), where ice can be warmed to temperatures allowing both rotational $(T \gtrsim 115 \mathrm{~K})$ and long-range translational $(T \gtrsim 120 \mathrm{~K})$ diffusion of heavy molecules. Under such conditions, molecules or/and radicals observed in IR spectra of ices (Dartois 2005) can effectively form iCOMs in the volume of the icy mantles.

On the one hand, when interstellar grains are in a certain vicinity of a protostar, their icy mantles tend to crystallize to a thermodynamically stable cubic lattice, but on the other hand, the ice becomes amorphous due to bombardment by high-energy electrons (Dubochet \& Lepault 1984) and ions (Baratta et al. 1991; Moore \& Hudson 1992; Dartois et al. 2015), or due to irradiation by UV photons (Kouchi \& Kuroda 1990; Leto \& Baratta 2003). Depending on the location of dust grains with respect to the protostar, the radical creation by radiolysis and photochemistry and the reactivity driven by the structural changes can provide an extremely efficient combination for creating iCOMs 
in the icy mantles. In this context, for the theoretical description of ice chemistry in protoplanetary disks, it can be crucial to account for the episodic changes in the ice surface-to-volume ratio caused by the temperature variations.

By lifting the constraints imposed by the conventional reaction-diffusion paradigm on the molecular transport in the bulk ice, the reactivity driven by the structural changes opens promising new ways to produce iCOMs in interstellar environment. In terms of the reaction rates and yields, this mechanism can provide a dramatic enhancement in comparison with the conventional transport models. Further experiments are certainly needed to carefully investigate the effect of different initial ice morphologies and thermal histories on the proposed reactivity mechanism, in order to implement this in the gas-grain chemistry models.

Acknowledgements. This work was supported by the Programme Nationa Physique et Chimie du Milieu Interstellaire (PCMI) of CNRS/INSU with INC/INP co-funded by CEA and CNES

\section{References}

Aikawa, Y., Ohashi, N., \& Herbst, E. 2003, ApJ, 593, 906

Bacmann, A., Taquet, V., Faure, A., Kahane, C., \& Ceccarelli, C. 2012, A\&A, 541, L12

Baragiola, R. A. 2003, Planet. Space Sci., 51, 953

Baratta, G. A., Leto, G., Spinella, F., Strazzulla, G., \& Foti, G. 1991, A\&A, 252, 421

Belloche, A., Müller, H. S. P., Garrod, R. T., \& Menten, K. M. 2016, A\&A, 587, A91

Borget, F., Müller, S., Grote, D., et al., 2017, A\&A, 598, A22

Bossa, J. B., Theulé, P., Duvernay, F., Borget, F., \& Chiavassa, T. 2008, A\&A, 492, 719

Bossa, J.-B., Isokoski, K., Paardekooper, D. M., et al. 2014, A\&A, 561, A136

Bowron, D. T., Finney, J. L., Hallbrucker, A., et al. 2006, J. Chem. Phys., 125, 194502

Caselli, P., \& Ceccarelli, C. 2012, A\&ARv, 20, 56

Collings, M. P., Anderson, M. A., Chen, R., et al. 2004, MNRAS, 354, 1133

Dartois, E. 2005, Space Sci. Rev., 119, 293

Dartois, E., Augé, B., Boduch, P., et al. 2015, A\&A, 576, A125

Davies, J. K., Roush, T. L., Cruikshank, D. P., et al. 1997, Icarus, 127, 238

de Barros, A. L. F., da Silveira, E. F., Rothard, H., Langlinay, T., \& Boduch, P. 2014, MNRAS, 443, 2733

Dubochet, J., \& Lepault, J., 1984, J. Phys., 45, 85

Faure, M., Quirico, E., Faure, A., et al. 2015, Icarus, 261, 14

Finney, J. L., Hallbrucker, A., Kohl, I., Soper, A. K., \& Bowron, D. T., 2002 Phys. Rev. Lett., 88, 225503

Fraser, H. J., Collings, M. P., McCoustra, M. R. S., \& Williams, D. A. 2001, MNRAS, 327, 1165
Ghesquière, P., Mineva, T., Talbi, D., et al. 2015, Phys. Chem. Chem. Phys., 17, 11455

Gibb, E. L., Whittet, D. C. B., Boogert, A. C. A., \& Tielens, A. G. G. M. 2004, ApJs, 151, 35

Gladstone, G. R., Stern, S. A., Ennico, K., et al. 2016, Science, 351, 8866

Hagen, W., Tielens, A. G. G. M., \& Greenberg, J. M. 1983, A\&As, 51, 389

Hasegawa, T. I., Herbst, E., \& Leung, C. M. 1992, ApJs, 82, 167

Hill, R. C., Mitterdorfer, C., Youngs, T. G. A., et al. 2016, Phys. Rev. Lett., 116, 215501

Isokoski, K., Bossa, J.-B., Triemstra, T., \& Linnartz, H. 2014, Phys. Chem. Chem. Phys., 16, 3456

Jenniskens, P., Blake, D. F., Wilson, M. A., \& Pohorille, A. 1995, ApJ, 455, 389

Johari, G. P. 1991, J. Phys. Chem., 95, 6849

Karssemeijer, L. J., Ioppolo, S., van Hemert, M. C., et al. 2014, ApJ, 781, 16

Kerkhof, O., Schutte, W. A., \& Ehrenfreund, P. 1999, A\&A, 346, 990

Kouchi, A., \& Kuroda, T. 1990, Nature, 344, 134

Kouchi, A., \& Sirono, S.-i. 2001, Geophys. Res. Lett., 28, 827

Lauck, T., Karssemeijer, L., Shulenberger, K., et al. 2015, ApJ, 801, 118

Leto, G., \& Baratta, G. A. 2003, A\&A, 397, 7

Livingston, F. E., Smith, J. A., \& George, S. M. 2002, J. Phys. Chem. A, 106, 6309

Mate, B., Rodriguez-Lazcano, Y., \& Herrero, V. J. 2012, Phys. Chem. Chem. Phys., 14, 10595

May, R. A., Smith, R. S., \& Kay, B. D. 2012, Phys. Chem. Chem. Phys., 3, 327

May, R. A., Smith, R. S., \& Kay, B. D. 2013, J. Chem. Phys., 138, 104501

Mayer, E., \& Pletzer, R., 1986, Nature, 319, 298

Mispelaer, F., Theulé, P., Aouididi, H., et al. 2013, A\&A, 555, A13

Mitchell, E. H., Raut, U., Teolis, B. D., \& Baragiola, R. A. 2016, Icarus, 285, 291

Moore, M. H., \& Hudson, R. L. 1992, ApJ, 401, 353

Noble, J. A., Congiu, E., Dulieu, F., \& Fraser, H. J. 2012, MNRAS, 421, 768

Noble, J. A., Theulé, P., Duvernay, F., et al. 2014, Phys. Chem. Chem. Phys., 16, 23604

Oba, Y., Watanabe, N., Hama, T., et al. 2012, ApJ, 749, 67

Quirico, E., Moroz, L. V., Schmitt, B., et al. 2016, Icarus, 272, 32

Raut, U., Famà, M., Teolis, B. D., \& Baragiola, R. A. 2007, J. Chem. Phys., 127, 204713

Sandford, S. A., \& Allamandola, L. J. 1990, ApJ, 355, 357

Smith, R. S., Huang, C., \& Kay, B. D. 1997a, J. Phys. Chem. B, 101, 6123

Smith, R. S., Huang, C., Wong, E. K. L., \& Kay, B. D. 1997b, Phys. Rev. Lett., 79, 909

Smith, R. S., Matthiesen, J., Knox, J., \& Kay, B. D. 2011, J. Phys. Chem. A, 115, 5908

Stechauner, G., \& Kozeschnik, E., 2014, J. Mater. Eng. Perform., 23, 1576

Swain, M. R., Tinetti, G., Vasisht, G., et al. 2009, ApJ, 704, 1616

Theulé, P., Duvernay, F., Danger, G., et al. 2013, Adv. Space Res., 52, 1567

Tielens, A. G. G. M., \& Hagen, W. 1982, A\&A, 114, 245

Viti, S., Collings, M. P., Dever, J. W., McCoustra, M. R. S., \& Williams, D. A. 2004, MNRAS, 354, 1141

Wu, Y. C., Jiang, J., Wang, S. J., Kallis, A., \& Coleman, P. G., 2011, Phys. Rev. B 84, 064123

Yuan, C., Smith, R. S., \& Kay, B. D. 2016, Surf. Sci., 652, 350

Zhitnikov, R. A., \& Dmitriev, Y. A. 2002, A\&A, 386, 1129 